\title{
Psoriatic arthritis: is it time to treat-to-target or target to treat?
}

\author{
Ennio Lubrano $^{1} \cdot$ Fabio Massimo Perrotta ${ }^{1}$
}

Received: 8 October 2017 / Accepted: 17 October 2017 / Published online: 24 October 2017

(C) International League of Associations for Rheumatology (ILAR) 2017

\begin{abstract}
Treat-to-target recommendations provide physicians a useful strategy to reduce the probability of disease progression and in general to manage PsA patients. However, it could be difficult to apply in real life, since PsA is a very heterogeneous disease with different manifestations to be treated. In fact, depending on the patient's expectation, some aspects of the disease could be prominent or not, beyond the mere achievement of the target of remission or low-disease activity. In this context, the concept of a "target to treat" strategy should be taken in to account.
\end{abstract}

Keywords Outcome $\cdot$ Psoriatic arthritis $\cdot$ Remission . Treat-to-target

\section{Introduction}

Psoriatic arthritis (PsA) is a chronic and systemic disease characterized by association of psoriasis and arthritis belonging to the group of spondyloarthritis (SpA) [1]. Clinical involvement of PsA is multifaceted, with the presence of enthesitis, dactylitis, and peripheral and axial joint manifestations as well as extra-articular involvement (psoriasis, uveitis, inflammatory bowel diseases, and cardiovascular) [1,2]. Biologic and targeted synthetic drugs radically changed the treatment of SpAs and, in particular in PsA, the new active agents (antiTNF, anti-IL-12/23, anti-IL-17, PDE4 inhibitors)

Ennio Lubrano

enniolubrano@hotmail.com

1 Dipartimento di Medicina e di Scienze della Salute "Vincenzo Tiberio", Università degli studi del Molise, Via Giovanni Paolo II, C/da Tappino, 86100 Campobasso, Italy demonstrated their efficacy and effectiveness in all manifestations of the disease with the possibility to induce a state of low-disease activity and to achieve the clinical remission [3-9]. The latter could be defined as a lack of disease activity in all disease domains. Thus, in 2014, Smolen and colleagues first developed the concept of treat-to-target (T2T) strategy to be applied in SpA [10]. This concept, originally developed for chronic diseases such as diabetes, has already been successfully implemented for rheumatoid arthritis (RA) and requires a strategic approach with regular assessment of disease activity that drives therapeutic adaptations or changes of drugs to achieve a specific target (low-disease activity or remission) [6]. In RA, the concept of remission is strictly linked to the number of tender and swollen joints, but, in SpA and PsA, this concept is different. In fact, in these conditions, remission or low-disease activity was identified as the potential "target" in a T2T strategy; however, due to the complexity of the disease, several composite indices were proposed to identify disease activity status and to assess a condition of low-disease activity and remission that encompass all spectrums of the disease [10, 11]. The Disease Activity Score for Psoriatic Arthritis (DAPSA) and the Minimal Disease Activity (MDA) indices were, therefore, proposed in the recent $\mathrm{T} 2 \mathrm{~T}$ recommendation $[12,13]$.

\section{Treat-to-target or which target?}

MDA is a composite measure created specifically for patients with PsA that encompasses many clinically important aspects: arthritis, psoriasis, enthesitis, pain, patient-assessed global disease activity, and physical function [12]. Patients achieving MDA are more likely to have a better radiologic outcome, because the achievement of sustained MDA (defined as MDA for over 12 months at consecutive clinic visits) was 
found to reduce progression of radiographic joint damage over a 3-year period [14]. The MDA criteria were used in the TICOPA (Tight Control of PsA) study, the only randomized study to date addressing whether treating to target using a prespecified target can improve outcome in PsA [15]. However, MDA is achieved if a patient fulfills 5/7 criteria, and thus, MDA might therefore not be able to "weigh" some disease components and not being able to capture some targets of the disease. In fact, recently Marin et al. demonstrated that patients with PsA fulfilling the MDA criteria do not have swollen and tender joints, but have active skin involvement [16]. Furthermore, in our previous report, we demonstrated that up to one fourth of patients in remission or low-disease activity, using the various indices (DAPSA and MDA), had residual disease activity, mainly on the skin, as well as in other domains [17]. Given these data, the concept of residual disease activity and its implication on which "target to treat" should be considered, in a potential target to treat approach. In fact, the concept of residual disease activity in some domains could be important in patients' management. For instance, the management of axial disease is another aspect to be taken into account, since the composite scores are often borrowed from ankylosing spondylitis and it requires specific treatments [18]. DAPSA do not evaluate axial involvement and the same could be for MDA, even if we showed that MDA 5/7 is achievable in axial PsA [7]. The MDA 7/7 are more stringent criteria to be used in a T2T strategy and therefore could be more specific to identify a very low-disease activity [19]. However, in our experience, only about one fourth of PsA patients treated with biologics achieved MDA 7/7, leaving open the question if this instrument is not sensitive enough in real clinical practice [20]. Finally, although the T2T provides physicians a useful strategy to reduce the probability of disease progression and in general to manage PsA patients, it could be a difficult strategy in real life, since the heterogeneity of the disease at clinical assessment and, in turn, the identification of the most prominent manifestation to be treated. In other words, in real life we have, most of the time, the necessity to identify a target to be treated, namely, joint or skin or axial, more than a simple process aimed to achieve remission. In fact, depending on the patient's expectation, some aspects of the disease (such as skin involvement in area of the body not exposed in patients with prominent joint complaints) could be not important in the treatment strategy. With regard to this latter aspect, the management of PsA patients in different clinical settings, namely, dermatology or rheumatology outpatient clinics, has to take into account: physicians could have different perspectives on which target is the priority, with the possible bias to underestimate or overestimate some features of the disease. Moreover, a recent study demonstrated that, generally in a dermatology setting, patients with suspected PsA had a more delayed diagnosis, and the mean time from diagnosis to both csDMARDs and bDMARDs treatment was longer in respect to a rheumatology setting [21]. In this context, a target to treat strategy might be useful to apply: physician have to select the target, (enthesis, joints, skin, nail, axial) and choose the treatment strategy according to patient's perspective and complaints. If residual disease activity persists in some domains, a different treatment strategy could be applied. Some evidences suggest that the addition of csDMARDs to anti-TNF seems to be a safe and effective treatment for patients with PsA with uncontrolled cutaneous disease. In these patients, this therapeutic approach might be an alternative option to switching to other agents [22]. Obviously, further studies are needed in this field.

In conclusion, T2T recommendations deal with a general approach to treating PsA and are very useful to guide physicians in the management of PsA patients. However, the complexity of the disease may require a different approach taking into account the prominent manifestations, patients perspective, long-term health-related quality of life and social participation, control of articular and skin signs and symptoms, prevention of structural damage, and normalization or preservation of function. In this way, it might be important to identify a "target" to treat rather than the mere induction of remission or low-disease activity status.

\section{Compliance with ethical standards}

Disclosures None.

\section{References}

1. Ritchlin CT, Colbert RA, Gladman DD (2017) Psoriatic arthritis. N Engl J Med 376:957-970. https://doi.org/10.1056/ NEJMra1505557

2. Palazzi C, D' Angelo S, Lubrano E, Olivieri I (2008) Aortic involvement in ankylosing spondylitis. Clin Exp Rheumatol 26(3 Suppl 49):S131-S134

3. Spadaro A, Lubrano E, Marchesoni A, D'Angelo S, Ramonda R, Addimanda $\mathrm{O}$ et al (2013) Remission in ankylosing spondylitis treated with anti-TNF- $\alpha$ drugs: a national multicentre study. Rheumatology (Oxford) 52:1914-1919. https://doi.org/10.1093/ rheumatology/ket249

4. Perrotta FM, Marchesoni A, Lubrano E (2016) Minimal disease activity and remission in psoriatic arthritis patients treated with anti-TNF- $\alpha$ drugs. J Rheumatol 43:350-355. https://doi.org/10. 3899/jrheum. 150805

5. Lubrano E, Perrotta FM, Marchesoni A, D'Angelo S, Ramonda R, Addimanda $\mathrm{O}$ et al (2015) Remission in non-radiographic axial spondyloarthritis treated with anti-tumor necrosis factor- $\alpha$ drugs: an Italian multicenter study. J Rheumatol 42:258-263. https://doi. org/10.3899/jrheum.140811

6. Lubrano E, Perrotta FM, Kavanaugh A (2015) An overview of low disease activity and remission in psoriatic arthritis. Clin Exp Rheumatol 33(5 Suppl 93):S51-S54

7. Lubrano E, Parsons WJ, Perrotta FM (2016) Assessment of response to treatment, remission, and minimal disease activity in axial psoriatic arthritis treated with tumor necrosis factor inhibitors. J Rheumatol 43:918-923. https://doi.org/10.3899/jrheum.151404 
8. Perrotta FM, Lubrano E (2016) Subcutaneous anti-TNF alfa induced sustained minimal disease activity and remission in psoriatic arthritis patients: a retrospective study. Postgrad Med 128:693-696. https://doi.org/10.1080/00325481.2016.1220809

9. Lubrano E, Perrotta FM (2016) Beyond TNF inhibitors: new pathways and emerging treatments for psoriatic arthritis. Drugs 76:663-673. https://doi.org/10.1007/s40265-016-0557-4

10. Smolen JS, Braun J, Dougados M, Emery P, Fitzgerald O, Helliwell $\mathrm{P}$ et al (2014) Treating spondyloarthritis, including ankylosing spondylitis and psoriatic arthritis, to target: recommendations of an international task force. Ann Rheum Dis 73:6-16. https://doi. org/10.1136/annrheumdis-2013-203419

11. Smolen JS, Breedveld FC, Burmester GR, Bykerk V, Dougados M, Emery P et al (2016) Treating rheumatoid arthritis to target: 2014 update of the recommendations of an international task force. Ann Rheum Dis 75:3-15. https://doi.org/10.1136/annrheumdis-2015207524

12. Coates LC, Fransen J, Helliwell PS (2010) Defining minimal disease activity in psoriatic arthritis: a proposed objective target for treatment. Ann Rheum Dis 69:48-53

13. Schoels MM, Aletaha D, Alasti F, Smolen JS (2016) Disease activity in psoriatic arthritis (PsA): defining remission and treatment success using the DAPSA score. Ann Rheum Dis $75: 811-818$

14. Coates L, Helliwell P (2010) Validation of minimal disease activity criteria for psoriatic arthritis using interventional trial data. Arthritis Car Res 62:965-969

15. Coates LC, Moverley AR, McParland L, Brown S, Navarro-Coy N, O'Dwyer JL et al (2015) Effect of tight control of inflammation in early psoriatic arthritis (TICOPA): a UK multicentre, open-label, randomised controlled trial. Lancet 386:2489-2498. https://doi. org/10.1016/S0140-6736(15)00347-5

16. Marin J, Acosta Felquer ML, Ferreyra Garrot L, Ruta S, Rosa J, Soriano ER (2016) Patients with psoriatic arthritis fulfilling the minimal disease activity criteria do not have swollen and tender joints, but have active skin. J Rheumatol 43:907-910. https://doi. org/10.3899/jrheum.151101

17. Lubrano E, De Socio A, Perrotta FM (2017) Comparison of composite indices tailored for psoriatic arthritis treated with csDMARD and bDMARD: a cross-sectional analysis of a longitudinal cohort. J Rheumatol 44:1159-1164. https://doi.org/10.3899/jrheum.170112

18. Nash P, Lubrano E, Cauli A, Taylor WJ, Olivieri I, Gladman DD (2014) Updated guidelines for the management of axial disease in psoriatic arthritis. J Rheumatol 41:2286-2289. https://doi.org/10. 3899/jrheum.140877

19. Coates LC, Helliwell PS (2016) Defining low disease activity states in psoriatic arthritis using novel composite disease instruments. J Rheumatol 43:371-375

20. Lubrano E, Perrotta FM (2016) Defining low disease activity states in psoriatic arthritis using novel composite disease instruments. J Rheumatol 43:1765-1766. https://doi.org/10.3899/jrheum. 160386

21. Boehncke $\mathrm{WH}$ et al. Clinical specialty setting as a determinant for disease management in patients with psoriatic arthritis: an interim analysis of the cross-sectional observational study for presentation at the 2017 ACR/ARHP Annual Meeting, to be held in San Diego, CA, November 3-8

22. D'Angelo S, Cutro MS, Lubrano E, Leccese P, Mennillo GA, Ferrara N et al (2010) Combination therapy with ciclosporin and etanercept in patients with psoriatic arthritis. Ann Rheum Dis 69: 934-935. https://doi.org/10.1136/ard.2009.119446 